\title{
THE SYNERGY BETWEEN THE LAT AND GBM IN GLAST'S STUDY OF GAMMA-RAY BURSTS
}

\author{
David L. Band \\ CRESST/UMBC \\ Code 661, NASA/GSFC, Greenbelt, MD 20771
}

\begin{abstract}
Using semi-analytic calculations I characterize the gamma-ray bursts to which GLAST's LAT and GBM detectors will be sensitive. The thresholds of both instruments are at approximately the same $v f_{v} \propto E^{2} N(E)$ values, i.e., the thresholds can be connected by an $E^{-2}$ spectrum. Therefore simultaneous detections by both instruments will be biased towards spectral components flatter than $E^{-2}$.
\end{abstract}

Keywords: Gamma-ray bursts

PACS: $95.55 . \mathrm{Ka} ;$ 95.85.Pw; $98.70 . \mathrm{Rz}$

GLAST is expected to discover new gamma-ray burst temporal and spectral components that were only hinted at by the observations of the Compton Gamma-Ray Observatory's EGRET and BATSE detectors. GLAST's burst studies will be enhanced by the synergy between the Large Area Telescope (LAT; $<20 \mathrm{MeV}$ to $>300 \mathrm{GeV}$ ) and the GLAST Burst Monitor (GBM; $8 \mathrm{keV}$ to $30 \mathrm{MeV}$ ). Between these two detectors GLAST may observe burst spectra covering 7 energy decades; the GBM's field-of-view (FOV) covers the LAT's large FOV totally. Here I use semi-analytic calculations to characterize the bursts to which each detector will be sensitive.

EGRET indicated that some bursts' $\sim 1 \mathrm{GeV}$ emission was not merely an extrapolation of the 'prompt' $\sim 100 \mathrm{keV}$ emission observed by detectors such as BATSE and Swift. [6] However, before GLAST's launch we have little detailed guidance as to what to expect, and therefore my calculations use extrapolations from the $\sim 100 \mathrm{keV}$ observations; at a minimum, these calculations indicate the constraints that can be placed on the correlations between the LAT and GBM energy bands. Thus, in my analysis I assume a single spectral component in both the GBM and LAT bands which is described by the 'Band Function, [4]' a smoothly broken power law: a low energy power law, $E^{\alpha}$ (typically $\alpha \sim-1$ ); a high energy power law, $E^{\beta}$ (typically $\beta<-2$ ); a break between these two power laws characterized by $E_{p}$, the energy of peak of $E^{2} N(E) \propto v f_{v}$; and the normalization, $F_{T}$, the $1-1000 \mathrm{keV}$ flux. The expectations (e.g., spectrum and burst rate) for the GBM are based on BATSE,[1] which had an energy band similar to the GBM's NaI detectors.

The GBM will detect bursts with a rate trigger, which searches the detector rates for a statistically significant increase. The rates from the different $\mathrm{NaI}$ detectors are binned continuously in energy range $\Delta E$ and accumulation time $\Delta t$ bins. A count rate increase of $>4.5 \sigma$ in the bins from $2 \mathrm{NaI}$ detectors will be required for a trigger. For a given set of spectral parameters $\alpha, \beta$, and $E_{p}$, the peak value of $F_{T}$ (when integrated over $\Delta t$ ) will determine whether the burst is detected. Therefore the GBM's sensitivity is the threshold value of $F_{T}$ for a given set of spectral parameters. The lower set of curves on Figure 1 shows the threshold $F_{T}$ over $\Delta t=1 \mathrm{~s}$ as a function of $E_{p}$, holding $\alpha=-1$ fixed for $\beta=-2$ (solid curve), -2.5 (dashed curve), and -3 (dot-dashed curve). Note that $F_{T}$ at a given $E_{p}$ is not the detector sensitivity at a photon energy equal to $E_{p}$. The $E_{p}$ and peak $F_{T}$ for a sample of BATSE bursts are shown by the dots; [7] a large fraction of this sample is below the GBM trigger threshold for $\Delta t=1 \mathrm{~s}$ (see [2]).

In my GBM calculations I use a preliminary 'direct' response function, i.e., with no scattering off the spacecraft or Earth's atmosphere. The background is modeled on the BATSE backgrounds. Different $\triangle E$ are used to maximize the GBM's sensitivity to bursts with low and high $E_{p}$. The most effective $\Delta E$ for a given burst depends on the spectral shape of the burst and the background.

For comparison, I show on Figure 1 (upper set of curves) the values of $F_{T}$ and $E_{p}$ for spectra that, when extrapolated to the LAT energy band, would result in 5 LAT counts in $\Delta t=1 \mathrm{~s}$ (i.e., 5 photons would be detected). Given the low LAT background, a statistically significant detection in $1 \mathrm{~s}$ will require of order 5 counts. Again, $\alpha=-1$, and $\beta=-2$ (solid curve), -2.5 (dashed curve), and -3 (dot-dashed curve). For this analysis I use a more inclusive set of cuts that increases the effective area at the expense of greater background (the ' $\mathrm{DC} 2$ ' analysis classes A and B).

This analysis assumed that $\Delta t=1 \mathrm{~s}$. However, gamma-ray burst durations are both shorter and longer than $1 \mathrm{~s}$. The GBM trigger will use a set of $\Delta t$ that will increase the sensitivity to both long and short bursts (see $[1,2,3]$ for the 


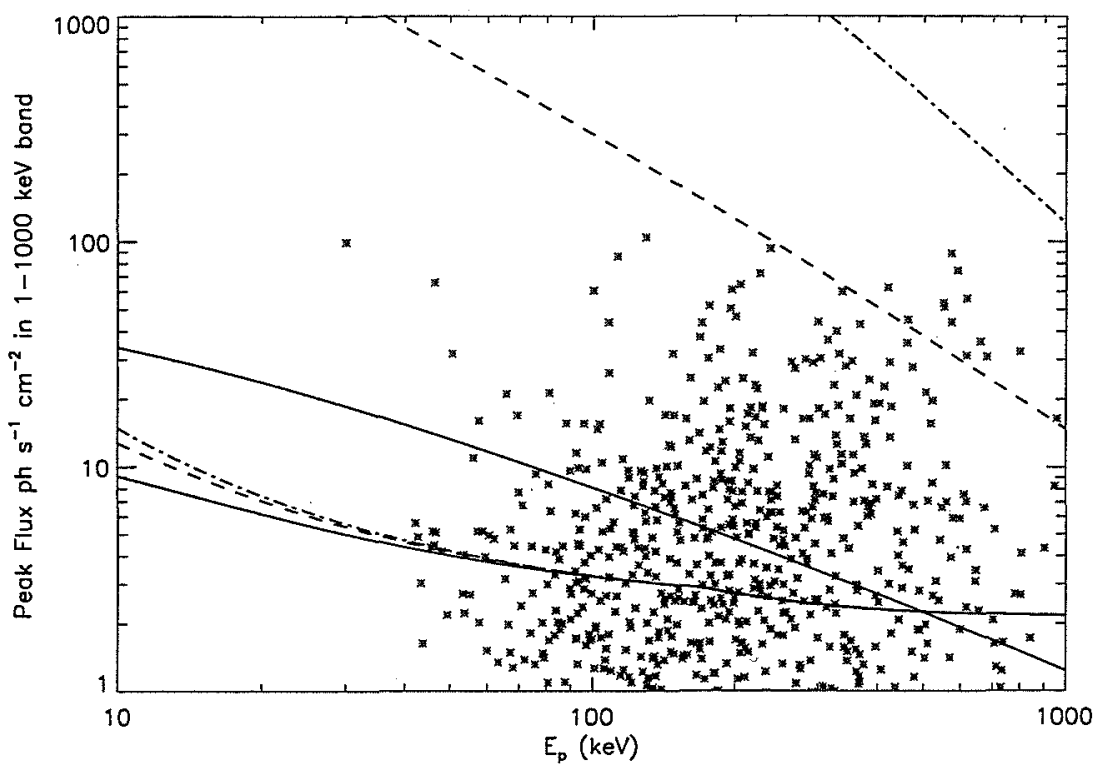

FIGURE 1. Comparison of LAT (upper set of curves) and GBM (lower set of curves) sensitivities as a function of $E_{p}$ for $\Delta t=1 \mathrm{~s}$. For each set of curves the low energy spectral index $\alpha=-1$ and the high energy spectral index $\beta=-2$ (solid curves), -2.5 (dashed curves) and -3 (dot-dashed curves). The dots result from fits to a set of BATSE bursts. See the text for further details.

dependence on $\Delta t$ ). Similarly, the LAT count data can be searched for burst emission on different timescales; statistical significance will depend on both timescale and photon energy.

Nonetheless, Figure 1 shows that the GBM and LAT are well matched for bursts with $\beta=-2$, i.e, constant $v f_{v} \propto$ $E^{2} N(E)$. LAT bursts with $\beta<-2.5$ will be brighter in the GBM band than most of the bursts that BATSE observed, and therefore rare. Thus there will be a bias towards LAT bursts with $\beta=-2$. These conclusions assume that the GBM and LAT observe the same spectral component. Additional spectral and temporal components are expected, $[6,5]$ and thus LAT emission may be detected even when $\beta<-2.5$ for the GBM spectra. Conversely, in general the non-detection of LAT counts will be relevant when $\beta>-2.5$ for the GBM spectra. Thus this type of analysis can be used to understand the burst populations GLAST's detectors detect, and do not detect.

\section{ACKNOWLEDGMENTS}

I thank members of the LAT GRB science working group and of the GBM instrument team for data, information, assistance and comments.

\section{REFERENCES}

1. D. Band, Ap. J., 578, 806, 2002.

2. D. Band, Ap. J., 588, 945, 2003.

3. D. Band, Ap. J., 644, 378, 2006.

4. D. Band, et al., Ap. J., 413, 281, 1993.

5. M. Gonzalez, et al., Nature, $424,749,2003$ ).

6. K. Hurley, et al., Nature, 372, 652, 1994.

7. R. Mallozzi, R., et al., "Gamma-Ray Burst Spectra and the Hardness-Intensity Correlation," in Gamma-Ray Bursts, 4th Huntsville Symposium, edited by C. Meegan, R. Preece and T. Koshut, AIP Conference Proceedings 428, American Institute of Physics, New York, 1998, p. 273. 